\title{
HOMOLOGICAL DIMENSION OF ABELIAN GROUPS OVER THEIR ENDOMORPHISM RINGS ${ }^{1}$
}

\author{
FRED RICHMAN AND ELBERT A. WALKER
}

\begin{abstract}
The projective dimension of an abelian group with torsion reduced part, as a module over its endomorphism ring, is determined. In particular, a group of projective dimension 2 is exhibited.
\end{abstract}

Any abelian group $G$ may be considered as a module over its endomorphism ring $E$. It then is natural to study the properties of $G$ as an $E$-module. In particular, you can try to compute the various homological dimensions of $G$. For example, in [6] we showed that a $p$-group $G$ is flat over $E$ (i.e. has weak dimension zero) unless its reduced part is bounded and its divisible part is nonzero. In [7] we characterized those $G$ which are injective over $E$. Douglas and Farahat [2] showed that if $G$ is torsion, then $\operatorname{dim}_{E} G \leqslant 1$, where $\operatorname{dim}_{E} G$ denotes the projective dimension of $G$ over $E$. Later [3] they showed that if $G$ is divisible, then $\operatorname{dim}_{E} G \leqslant 1$. In both of these papers they raised the question as to whether $\operatorname{dim}_{E} G \leqslant 1$ for all abelian groups $G$. The purpose of this paper is to determine the projective dimension of groups whose reduced part is torsion. This combines and generalizes the results in the two Douglas and Farahat papers and exhibits groups of projective dimension 2.

We begin with an observation of Douglas and Farahat $[2,1]$, a simplified version of a theorem in Northcott [5, Lemma 2, p. 139], and a (very slightly) generalized version of Douglas and Farahat's theorem about torsion groups, which we prove mainly for the convenience of the reader.

Lemma 1. Let $G$ be an abelian group, $E$ its endomorphism ring, and $A$ a summand of $G$. Then $\operatorname{Hom}(A, G)$ is a projective E-module.

Proof. Let $e \in E$ be a projection on $A$. Then $\operatorname{Hom}(A, G) \cong E e$ is a summand of $E$.

Lemma 2. Let $A_{0} \subseteq A_{1} \subseteq A_{2} \subseteq \cdots$ be $R$-modules such that $\operatorname{dim}_{R} A_{0} \leqslant n$ and $\operatorname{dim}_{R} A_{i} / A_{i-1} \leqslant n$ for $i=1,2, \cdots$. Then $\operatorname{dim}_{R} \cup A_{i} \leqslant n$.

Proof. See Northcott [5, Lemma 2, p. 139] or fill in the details in the following sketch. If $n=0$, then $\cup A_{i} \cong A_{0} \oplus A_{1} / A_{0} \oplus A_{2} / A_{1} \oplus \cdots$ so the lemma is true. If $n>0$, map projectives $P_{i}$ onto the $A_{i} / A_{i-1}$ and lift to $A_{i}$. Then $\bigoplus P_{i}$ maps onto $\cup A_{i}$ and the kernel has dimension less than $n$ by induction.

Received by the editors January 27, 1975.

AMS (MOS) subject classifications (1970). Primary 18G20, $20 \mathrm{~K} 40$.

1 This research was supported in part by NSF Grant GP-28379. 
In particular, if $A_{0} \subseteq A_{1} \subseteq A_{2} \subseteq \cdots$ are all projective, then $\operatorname{dim} \cup A_{i}$ $\leqslant 1$. This is easily proved directly by mapping $\bigoplus A_{i}$ onto $\cup A_{i}$ and observing that the kernel is isomorphic to $\bigoplus A_{i}$.

Lemma 3 (Douglas And Farahat). Let $G$ be an abelian group, $E$ its endomorphism ring, and $H$ a pure, fully invariant, torsion subgroup of $G$. Then $\operatorname{dim}_{E} H \leqslant 1$.

Proof. Since $H$ is the direct sum of its primary subgroups, which are invariant under $E$, we may assume that $H$ is $p$-primary. Let $N=\{n: H$ has a cyclic summand of order $n\}$. Note that a cyclic summand of $H$ is also a summand of $G$. If $N$ is infinite then

$$
H=\bigcup_{n \in N} H[n]
$$

and since $H[n] \cong \operatorname{Hom}(C, G)$ where $C$ is a cyclic summand of order $n$, we have that $H$ is the union of a countable chain of $E$-projectives, by Lemma 1, so $\operatorname{dim}_{E} H \leqslant 1$ by Lemma 2 .

If $N$ is finite, let $n$ be its largest element. Let $A_{i}=H\left[n p^{i}\right]$. Now $A_{0}=H[n]$ is $E$-projective as before. Since $H=B \oplus D$ where $n B=0$ and $D$ is divisible, the $E$-module $A_{i} / A_{i-1}$ is generated by any element $x$ in $D$ of order $n p^{i}$. So $A_{i} / A_{i-1} \cong E / I$ where $I=\left\{e \in E:\right.$ ex $\left.\in A_{i-1}\right\}$. Write $G=G_{1} \oplus G_{2}$ where $G_{1}$ is a rank-one summand containing $x$. Then $I=E e_{0}$ where $e_{0}$ is the identity map on $G_{2}$ and multiplication by $p$ on $G_{1}$. Since $E e_{0} \cong E$ is projective, we have $\operatorname{dim}_{E} A_{i} / A_{i-1} \leqslant 1$ and so $\operatorname{dim}_{E} H \leqslant 1$ by Lemma 2 .

The following lemma is a slight generalization of the characterization in [6] of $p$-groups that are projective over their endomorphism rings.

Lemma 4. Let $G$ be an abelian group, $E$ its endomorphism ring, and $H$ a pure, fully invariant, torsion subgroup of $G$. Then $H$ is E-projective if and only if every p-primary subgroup of $H$ is bounded.

Proof. We may assume that $H$ is $p$-primary. If $H$ is bounded, then $H$ is isomorphic to $\operatorname{Hom}(C, G)$ where $C$ is a cyclic summand of $H$ of maximum order. So $H$ is $E$-projective by Lemma 1 . Suppose $H$ is $E$-projective. Consider the map

$$
\phi: \operatorname{Hom}_{E}(H, E) \otimes_{E} H \rightarrow \operatorname{Hom}_{E}(H, H)
$$

given by $\phi(f \otimes h)(x)=f(x) h$. By the dual basis theorem [1, Proposition 3.1, p. 132], the image of $\phi$ contains elements fixing any finite subset of $H$, so $\phi$ is nonzero if $H$ is nonzero. If $H$ is unbounded, then $\operatorname{Hom}_{E}(H, H)$ is isomorphic to the $p$-adic integers (proof indentical to showing that the center of $\operatorname{Hom}_{Z}(H, H)$ is isomorphic to the $p$-adic integers, see [4, Theorem 108.3]). But $\operatorname{Hom}_{E}(H, E) \otimes_{E} H$ is torsion because $H$ is torsion. Thus $H$ must be bounded.

The next lemma generalizes Douglas and Farahat's result on divisible groups [3], using much the same argument as they do.

Lemma 5. Let $G$ be an abelian group, $E$ its endomorphism ring, and $H$ a pure, fully invariant subgroup of $G$. If the reduced part of $H$ is bounded, then $\operatorname{dim}_{E} H \leqslant 1$. 
Proof. If $H$ is torsion we are done by Lemma 3. Otherwise write

$$
H=L \oplus\langle x\rangle \oplus H^{\prime}
$$

where $L$ is rank-one, torsion free, divisible, and $x$ is a torsion element of maximum order. Choose $0 \neq y \in L$ and consider the $E$-map

$$
\phi: \operatorname{Hom}(L \oplus\langle x\rangle, G) \rightarrow H
$$

given by $\phi(f)=f(y+x)$. The kernel of $\phi$ is $\operatorname{Hom}((L \oplus\langle x\rangle) /\langle y+x\rangle, G)$ which is isomorphic to $\operatorname{Hom}(Q / Z, G)$ which is isomorphic to $\operatorname{Hom}(A, G)$ where $A$ is a divisible summand of $G$ containing one copy of each $Z\left(p^{\infty}\right)$ that occurs in $G$. Thus, by Lemma 1 , we have $\operatorname{dim}_{E} H \leqslant 1$.

THEOREM. Let $G$ be an abelian group, $E$ its endomorphism ring, and $H=T \oplus D$ a pure, invariant subgroup of $G$, with $T$ torsion reduced and $D$ divisible. Then

(1) $\operatorname{dim}_{E} H \leqslant 2$.

(2) $\operatorname{dim}_{E} H \leqslant 1$ if and only if $D$ is torsion $\operatorname{or} \operatorname{Hom}(T, D)$ is bounded.

(3) $\operatorname{dim}_{E} H=0$ if and only if $D$ is torsion free and $T_{p}$ (the p-primary part of $T$ ) is bounded for all $p$.

Proof. If $H$ is torsion, then (1) and (2) follow from Lemma 3, while (3) is Lemma 4. So we may assume that $H$ is not torsion and, hence, contains a summand of $G$ isomorphic to $Q$. Let $N=\{n: H$ has a cyclic summand of order $n\}$ and $P=\left\{p: H\right.$ has a cyclic summand of type $\left.Z\left(p^{\infty}\right)\right\}$. The "if" half of (3) follows from Lemma 4 and the observation that if $D$ is torsion free, then $D \cong \operatorname{Hom}(Q, G)$ is $E$-projective. The "if" half of (2) follows from Lemmas 3 and 5 since $H$ is the direct sum of the invariant subgroups $\sum_{p \notin P} T_{p}$ and $D+\Sigma_{p \in P} T_{p}$. To complete the proof we consider the projective resolution

$$
0 \rightarrow K \rightarrow \bigoplus_{n \in N} H[n] \oplus \operatorname{Hom}(Q, G) \rightarrow H \rightarrow 0
$$

where $\bigoplus a_{i}+f$ maps to $\sum a_{i}+f(1)$. Let

$$
M=K \cap \operatorname{Hom}(Q, G)=\{f \in \operatorname{Hom}(Q, G): f(1)=0\} .
$$

Then $M \cong \operatorname{Hom}\left(\sum_{p \in P} Z\left(p^{\infty}\right), G\right)$ is algebraically compact, torsion free, reduced (see [4, Proposition 44.3]), and $E$-projective. The torsion subgroup of $K$ is

$$
K_{t}=K \cap \bigoplus_{n \in N} H[n]
$$

and is $E$-projective since it is the kernel of a projective resolution of $\Sigma H[n]$, which is the union of a countable chain of projectives. Projecting $K$ into $\operatorname{Hom}(Q, G)$ shows that

$$
\frac{K}{K_{t}} \cong\left\{f \in \operatorname{Hom}(Q, G): f(1) \in \sum_{n \in N} D[n]\right\}=\bigcup_{n \in N} \frac{M}{n}
$$

Since $M / n \cong M$ is $E$-projective, $\operatorname{dim}_{E} K / K_{t} \leqslant 1$ and, hence, $\operatorname{dim}_{E} K \leqslant 1$. This proves (1).

If $H$ is $E$-projective, then $H$ is a summand of $\bigoplus_{n \in N} H[n] \oplus \operatorname{Hom}(Q, G)$, so $D$ must be torsion free. This, with Lemma 4, completes the proof of (3). 
Finally, suppose $\operatorname{dim}_{E} H \leqslant 1$, so $K$ is $E$-projective. Since

$$
\frac{K}{K_{t}} \cong \bigcup_{n \in N} \frac{M}{n}
$$

and $M$ is $E$-projective, we can map a direct sum of copies of $M$ and $K_{t}$ onto $K$. Since $K$ is $E$-projective, this map splits. But $M$ is algebraically compact, and $K_{t}$ is a direct sum of finite cyclic groups, so by a theorem of Warfield [8] we can write $K=\bigoplus C_{\alpha}$ where $C_{\alpha}$ is algebraically compact. Since $M$ is torsion free algebraically compact, we must have $M \cap C_{\alpha}=0$ for all $\alpha$ outside some finite set $F$. But $K / M$ is isomorphic to a subgroup of $\bigoplus_{n \in N} H[n]$ and so is torsion and reduced. Thus $C_{\alpha} / M \cap C_{\alpha}$ is bounded for each $\alpha$. Choose a nonzero integer $m$ such that $m \bigoplus_{\alpha \in F}^{\alpha} C_{\alpha} / M \cap C_{\alpha}=0$. Then $m K$ $=m\left(\bigoplus_{\alpha \in F} C_{\alpha} \oplus \bigoplus_{\alpha \in F} C_{\alpha}\right) \subseteq K_{t}+M$. But if $f \in \operatorname{Hom}(Q, G)$ and $f(1) \in$ $\sum_{n \in N} D[n]$, then $f(1)-f \in K$ so $m f(1)-m f \in K_{t}+M$. Since $K_{t}$ $\subseteq \bigoplus_{n \in N} H[n]$ and $M \subseteq \operatorname{Hom}(Q, G)$, we have

$$
m f \in M=\{f \in \operatorname{Hom}(Q, G): f(1)=0\} \text {. }
$$

Thus, $m f(1)=0$; hence, $m \sum_{n \in N} D[n]=0$ and so $m \operatorname{Hom}(T, D)=0$. This proves the "only if" half of (2) and completes the proof of the theorem.

In particular, there exist abelian groups of dimension 2 over their endomorphism rings. One such group is the direct sum of $Q$ and the groups $Z\left(p^{n}\right)$ for $1 \leqslant n \leqslant \infty$. The existence of groups of dimension 3 or greater remains an open question.

AdDED IN PROOF. In Monat. für Math. 80 (1975), Douglas and Farahat exhibit a group of infinite dimension.

\section{REFERENCES}

1. H. Cartan and S. Eilenberg, Homological algebra, Princeton Univ. Press, Princeton, N. J., 1956. MR17, 1040.

2. A. J. Douglas and H. K. Farahat, The homological dimension of an Abelian group as a module over its ring of endomorphisms, Monatsh. Math. 69(1965), 294-305. MR32 \#2473.

3. The homological dimension of an abelian group as a module over its ring of endomorphisms. II, Monatsh. Math. 76(1972), 109-111. MR47 \#3568.

4. L. Fuchs, Infinite abelian groups. Vols. I, II, Pure and Appl. Math., vol. 36, Academic Press, New York, 1970, 1973. MR41 \#333.

5. D. G. Northcott, An introduction to homological algebra, Cambridge Univ. Press, New York, 1960. MR22 \#9523.

6. F. Richman and E. A. Walker, Primary abelian groups as modules over their endomorphism rings, Math. Z. 89(1965), 77-81. MR32 \#2475.

7. . Modules over PID's that are injective over their endomorphism rings, Ring Theory, edited by R. Gordon. Academic Press, New York, 1972, pp. 363-372.

8. R. B. Warfield, Jr., An isomorphic refinement theorem for abelian groups, Pacific J. Math. 34(1970), 237-255. MR42 \#1896.

Department of Mathematics, New Mexico State University, Las Cruces, New Mexico 88003 